\title{
Solar influences on dynamical coupling between the stratosphere and troposphere
}

Article

Accepted Version

Haigh, J. D. and Blackburn, M. (2006) Solar influences on dynamical coupling between the stratosphere and troposphere. Space Science Reviews, 125 (1-4). pp. 331-344. ISSN 15729672 doi: https://doi.org/10.1007/s11214-006-9067-0 Available at https://centaur.reading.ac.uk/190/

It is advisable to refer to the publisher's version if you intend to cite from the work. See Guidance on citing.

Published version at: http://dx.doi.org/doi:10.1007/s11214-006-9067-0

To link to this article DOI: http://dx.doi.org/10.1007/s11214-006-9067-0

Publisher: Springer

Publisher statement: The original publication is available at www.springerlink.com

All outputs in CentAUR are protected by Intellectual Property Rights law, including copyright law. Copyright and IPR is retained by the creators or other copyright holders. Terms and conditions for use of this material are defined in the End User Agreement.

\section{www.reading.ac.uk/centaur}

\section{CentAUR}

Central Archive at the University of Reading

Reading's research outputs online 


\title{
SOLAR INFLUENCES ON DYNAMICAL COUPLING BETWEEN THE STRATOSPHERE AND TROPOSPHERE
}

\author{
J. D. HAIGH (j . haigh@imperial .ac .uk) \\ Blackett Laboratory, Imperial College London, UK \\ M. BLACKBURN (m . blackburn@rdg . ac . uk) \\ Centre for Global Atmospheric Modelling, University of Reading, UK
}

Received: ...; Accepted in final form: ...

\begin{abstract}
We use a simplified atmospheric general circulation model (AGCM) to investigate the response of the lower atmosphere to thermal perturbations in the lower stratosphere. The results show that generic heating of the lower stratosphere tends to weaken the sub-tropical jets and the tropospheric mean meridional circulations. The positions of the jets, and the extent of the Hadley cells, respond to the distribution of the stratospheric heating, with low latitude heating displacing them poleward, and uniform heating displacing them equatorward. The patterns of response to the low latitude heating are similar to those found to be associated with solar variability in previous observational data analysis, and to the effects of varying solar UV radiation in sophisticated AGCMs. In order to investigate the chain of causality involved in converting the stratospheric thermal forcing to a tropospheric climate signal we conduct an experiment which uses an ensemble of model spin-ups to analyse the time development of the response to an applied stratospheric perturbation. We find that the initial effect of the change in static stability at the tropopause is to reduce the eddy momentum flux convergence in this region. This is followed by a vertical transfer of the momentum forcing anomaly by an anomalous mean circulation to the surface, where it is partly balanced by surface stress anomalies. The unbalanced part drives the evolution of the vertically integrated zonal flow. We conclude that solar heating of the stratosphere may produce changes in the circulation of the troposphere even without any direct forcing below the tropopause. We suggest that the impact of the stratospheric changes on wave propagation is key to the mechanisms involved.
\end{abstract}

\section{Introduction}

The existence of signals of the 11-year solar cycle in meteorological fields of the lower atmosphere has been confirmed in analyses of observational data (see e.g. van Loon and Shea, 2000; Haigh, 2003, Gleisner and Thejll, 2003, Crooks and Gray 2005). The responses found are latitudinally non-uniform and locally larger than would be predicted from radiative forcing considerations alone (Haigh, 2001). Atmospheric general circulation models (AGCMs) are beginning to be able to reproduce some of these effects when forced by changes in solar ultraviolet radiation in the stratosphere (Haigh 1996, 1999; Shindell et al 1999; Matthes et al 2004) suggesting that changes in the stratosphere may exert some dynamical control on the troposphere. It is well known that planetary waves propagating upwards from the troposphere can influence the state of the stratosphere but the mechanisms whereby the stratosphere can influence the 
troposphere are not well established and form the subject of active current research (see Haynes, 2005 for a review).

In this paper we use a simplified AGCM to investigate how thermal perturbations to the stratosphere affect the climate of the troposphere. Our work is motivated by the desire to understand how solar variability influences climate but our results are applicable more generally to understanding stratosphere-troposphere coupling.

\section{Response of tropospheric zonal wind to solar variability}

In an earlier paper (Haigh et al, 2005) we demonstrated, using a multiple regression analysis, the existence of a statistically significant signal of solar 11year cycle variability in the lower atmosphere annual mean zonal winds of the National Centers for Environmental Prediction/National Center for Atmospheric Research (NCEP/NCAR) reanalysis dataset, provided by the Climate Diagnostics Center, Boulder, USA (http://www.cdc.noaa.gov/). A similar result, for northern hemisphere winter, is presented in Figure 1(d): the solar signal is seen to be composed of banded structures in mid-latitudes with the largest change, a reduction of over $1 \mathrm{~ms}^{-1}$, present on the equatorward sides of the midlatitude jet cores, and an increase, of a few tenths of $1 \mathrm{~ms}^{-1}$, on the poleward sides. By comparison with the mean zonal wind (Figure 1(c)) it can be seen that this represents, when the sun is more active, a weakening of the winter uppertropospheric sub-tropical jet, and a poleward shift of the mid-latitude jets in both hemispheres.
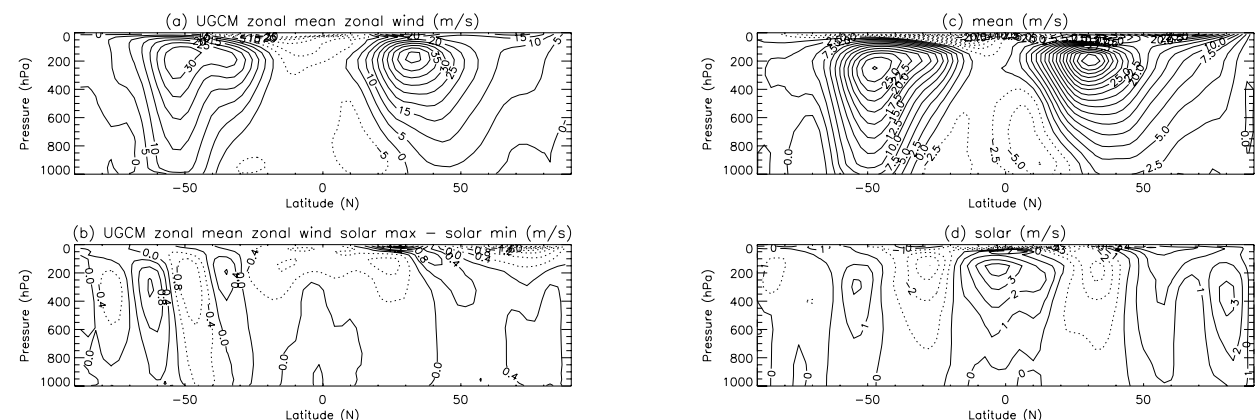

Figure 1. Zonal mean zonal wind (a) January mean from GCM; (b) Solar signal in January from GCM experiments; (c) DJF mean of NCEP reanalysis data 1979-2002; (d) Solar signal in DJF from multiple regression analysis of NCEP data.

Both the shape and the amplitude of this pattern are remarkably similar to those found in the GCM simulations of Haigh (1996, 1999), reproduced in Figure 1 (a) and (b). [Note, however, this GCM has some problems in the southern hemisphere producing a strong sub-tropical jet feature that is not found in observations for this 
season.] Using entirely different models, Larkin et al (2000) and Matthes et al (2004) find a similar tropospheric response. These model studies included the effects of changes in solar spectral irradiance where the largest direct radiative impact, due to enhanced solar UV, is found in the stratosphere. The GCMs also predicted a response to higher levels of solar UV in the tropospheric mean meridional circulation consisting of a weakening and expansion of the Hadley cells and poleward shift of the Ferrel cells.

The similarity of the signals found in the observational data and model runs is intriguing and, by the nature of the model experiments, suggests that changes in the stratosphere, introduced by modulation of solar ultraviolet radiation and ozone, are key. It does not, however, explain the mechanisms whereby such a change in tropospheric circulation is brought about by thermal perturbations to the stratosphere. In Section 3 we describe some experiments with a simplified GCM designed to elucidate some of these mechanisms. An initial discussion of the results of these runs was included in Haigh et al (2005); some of these are reviewed here, together with further analysis. In Section 4 we present results from a new experiment in which an ensemble of model spin-ups is used to investigate the mechanisms involved in producing the effects found in the perturbation experiments discussed in Section 3.

\section{Impact of stratospheric heating on the troposphere of a simplified GCM}

The model uses the spectral dynamical core described by Hoskins and Simmons (1975), modified to include the angular-momentum conserving vertical discretisation of Simmons and Burridge (1981), but retaining the original sigma coordinate. All experiments use a T42L15 resolution, denoting triangular spectral truncation at total wavenumber 42, equivalent to an equatorial grid spacing close to $475 \mathrm{~km}$ for the equivalent linear transform grid, and 15 levels between the surface and about $18.5 \mathrm{hPa}$. The model's climate is maintained using the linear forcing and drag scheme of Held and Suarez (1994), in place of the moist parameterisations of a sophisticated GCM. Orography is omitted. Temperature is relaxed towards the zonally symmetric equilibrium distribution shown in Figure 2(a) on a timescale which is 40 days above the boundary layer (pressure greater than about $700 \mathrm{hPa}$ ), but which reduces to 4 days at the equatorial surface. Rayleigh friction is also included in the boundary layer with a timescale of 1 day at the surface.

The dynamical response to the equilibrium structure is such as to cause adiabatic warming at mid-high latitudes and cooling at low latitudes so that, although the equilibrium temperature is uniform and constant above the tropopause, the model's temperature field (Figure 2(b)) shows a positive latitudinal temperature gradient there while in the troposphere the negative temperature gradients are reduced. The 
model's zonal wind structure is shown in Figure 2(c) to aid assessment of the results of the model experiments discussed below.
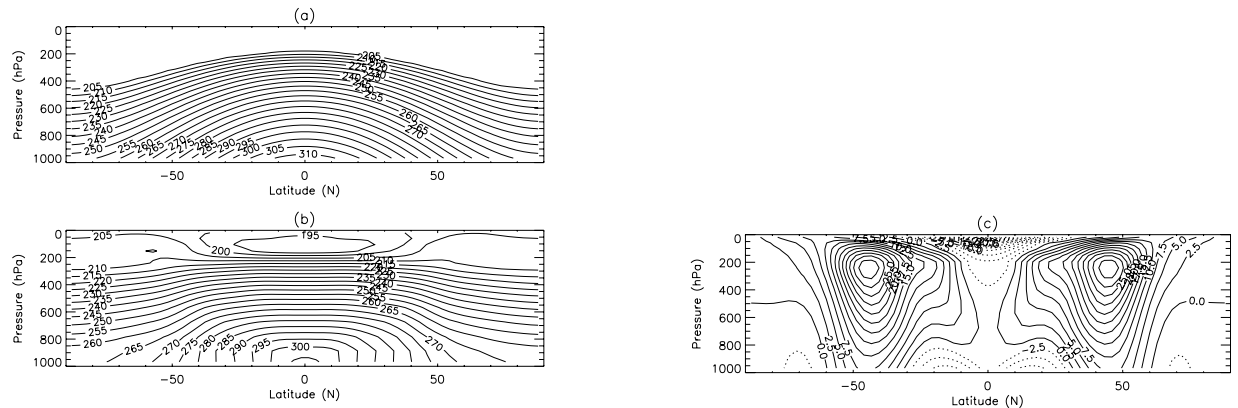

Figure 2 Simple GCM fields: (a) the prescribed equilibrium temperature (K); (b) the zonal mean temperature (K) for the control run $\mathrm{C}$ and $(\mathrm{c})$ the zonal mean zonal wind $\left(\mathrm{m} \mathrm{s}^{-1}\right)$ for run C.

The model climatology consists of a single jet which extends through the depth of the troposphere in the mid-latitudes of each hemisphere, maintained by fluxes of (heat and) momentum associated with the transient weather systems (shown later). The observed climate (Figure 1(c)) comprises an eddy-maintained jet in each hemisphere, and also a subtropical jet which is confined to the upper-troposphere of the winter hemisphere, and which is only partially separated from the eddymaintained jet. The existence of, and variations in, the subtropical jet involve moist tropical processes which have been idealised as a linear relaxation in the simplified model. The model results are therefore relevant only to stratospheric influences on the eddy-driven mid-latitude jets and not to other possible mechanisms such as changes in convective activity or the vertical propagation of stationary planetary waves, such as discussed by e.g. Rind and Balachandran (1995).

Experiments with the model have been designed to investigate the effects of perturbations to the temperature structure of the lower stratosphere. These are implemented by modifications of the equilibrium temperature distribution in the stratosphere as summarized in Table 1. Experiments U1 and U5 prescribe a uniform heating perturbation of $1 \mathrm{~K}$ and $5 \mathrm{~K}$ respectively, throughout the stratosphere (defined as the region of uniform temperature at the top of Figure 2(a)). In experiments E1 and $\mathrm{E} 5$ the equatorial stratosphere is warmed by $1 \mathrm{~K}$ and $5 \mathrm{~K}$ respectively but this is gradually reduced to zero increase at the poles. In no case is any perturbation applied within the troposphere. The structure of the perturbations is very simple, and the model runs are certainly not intended as direct simulations of solar effects, but we find that the results may be useful in interpreting the effects of solar perturbations to the real atmosphere.

The $U$ and $E$ experiments were each carried out with the two different amplitudes, of $1 \mathrm{~K}$ and $5 \mathrm{~K}$, in order to assess whether the patterns of response were independent of the magnitude of the forcing and to what extent the response scaled with the forcing. The statistical significance of the responses is estimated 
independently at each grid-point using a Student t-test taking into account the reduction in the number of degrees of freedom due to autocorrelations in the time series. The signals produced by the $5 \mathrm{~K}$ perturbations are much larger than the longterm variability in the fields so that it was necessary to continue these runs for only 1,000 days, after an initial spin-up period of 200 days. For the $1 \mathrm{~K}$ experiments, and the control, runs of 10,000 days were carried out. In all cases the effective number of data points has been doubled by using the results from the two hemispheres as independent simulations. This is possible because an analysis of the temporal correlations between equivalent points in the two hemispheres shows very low values (of magnitude $\sim 0.2$ ) across most of the hemisphere.

TABLE 1

Summary of experiments with the simplified GCM

\begin{tabular}{lll}
\hline Run ID & Length of run (days) & $\begin{array}{l}\text { Increase in stratospheric } \\
\text { equilibrium temperature }(\mathrm{K})\end{array}$ \\
\hline C & $2 \times 10,000$ & none \\
U1 & $2 \times 10,000$ & 1 \\
U5 & $2 \times 1,000$ & 5 \\
E1 & $2 \times 10,000$ & $\cos ^{2}$ (latitude) \\
E5 & $2 \times 1,000$ & $5 \cos ^{2}$ (latitude) \\
\hline
\end{tabular}

The perturbations in zonal mean zonal wind found in the simplified GCM experiments are presented in Figure 3. Banded structures appear in the troposphere in each case, despite the forcing having only been applied in the stratosphere. In runs U1 and U5 (Figure 3(a) and (b)) these appear as a band of weaker winds near $55^{\circ}$ and a band of stronger winds near $35^{\circ}$. This represents a weakening of the jet and an equatorward shift. This response is qualitatively similar (apart from a change in sign of both the forcing and response) to the results of Polvani and Kushner (2002) who presented results from a similar model used to study the impacts of polar lower stratospheric cooling. The response of the E experiments (Figures 3(c) and (d)) is different from the $U$ runs, with a negative band near $40^{\circ}$ flanked by positive bands near $25^{\circ}$ and $60^{\circ}$ respectively. These correspond to a weakening and latitudinal expansion, but mainly poleward shift, of the jets. This pattern is qualitatively similar to the solar signal found in the sophisticated GCM experiments and observational data analysis presented in Figure 1(b) and (d).

Clearly the responses seen in the simple model experiments U5 and E5 are much larger than those found to be due to solar influences in the data analysis. Experiments U1 and E1, using forcings which were a factor 5 smaller than U5 and E5, respectively, might be more appropriate for interpretation in this regard. However, as the patterns of response seen in the $1 \mathrm{~K}$ experiments are essentially identical to those of the corresponding $5 \mathrm{~K}$ runs it seems clear that the same processes are operating. On this basis, and for reasons of clarity and brevity, the analysis and interpretation of the data, below, is based on the $5 \mathrm{~K}$ experiments alone. 

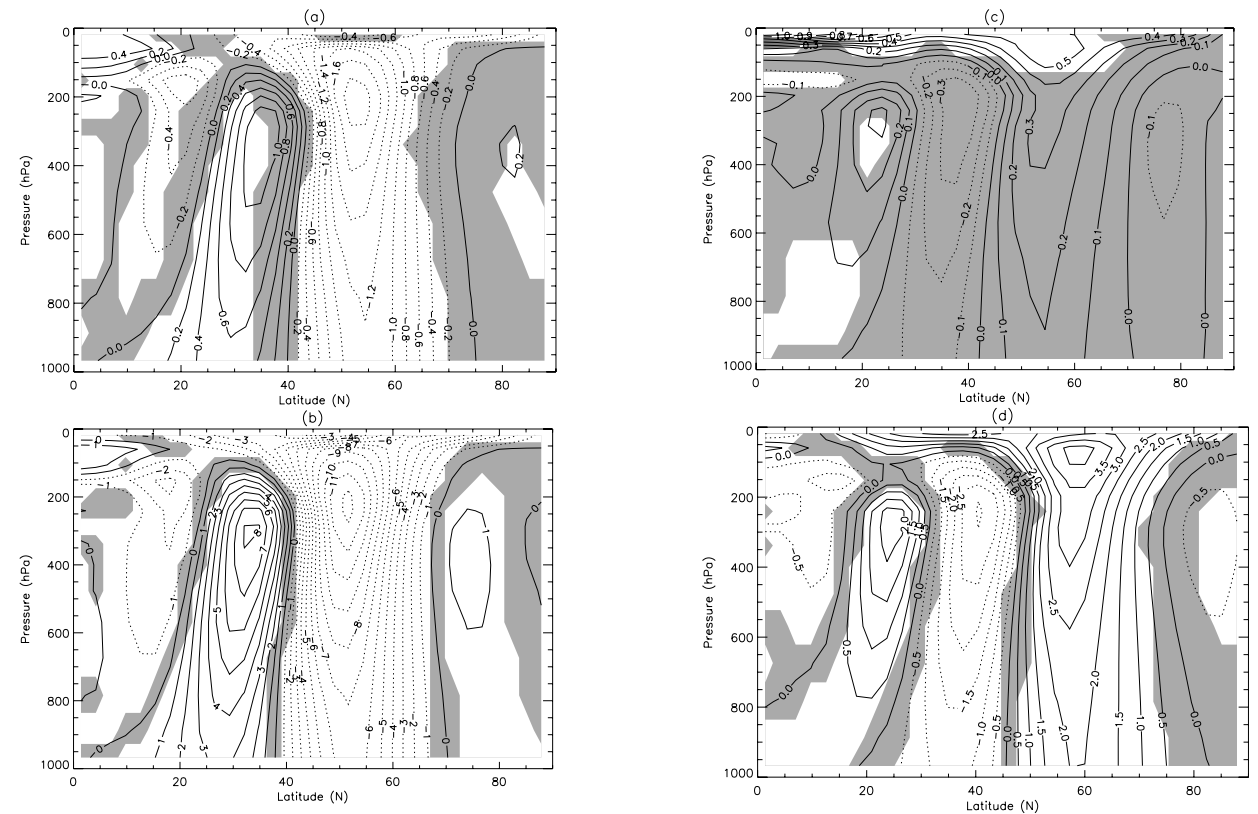

Figure 3. Difference from control run of zonal mean zonal winds in run (a) U1 (contour interval $\left.0.2 \mathrm{~ms}^{-1}\right)$, (b) U5 $\left(1 \mathrm{~ms}^{-1}\right)$, (c) E1 $\left(0.1 \mathrm{~ms}^{-1}\right)$ and (d) E5 $\left(0.5 \mathrm{~ms}^{-1}\right)$. Average of 2 hemispheres. Regions in which the signal does not reach the 95\% confidence level are shaded

The field of poleward eddy momentum flux for control run C, and the anomalies, relative to run $\mathrm{C}$, for runs $\mathrm{U} 5$ and $\mathrm{E} 5$ are shown in Figure 4. In the control run C there is poleward eddy momentum flux from the tropics into mid-latitudes, with maximum flux at $35^{\circ}$. The (vertically integrated) flux convergence in mid-latitudes acts as a forcing of westerly flow, maintaining the jet in Figure 2(c) against lowlevel drag, while the flux divergence in the subtropics acts as a forcing of easterly flow there, balancing the Coriolis force associated with the poleward outflow of the tropical Hadley circulation near the tropopause (shown in Figure 5(a)). The effect of stratospheric heating is to increase the static stability of the tropopause region and to lower the tropopause so that the wave flux convergence, and the poleward branch of the Hadley cell, are moved downwards. For run U5 the momentum flux is significantly weakened, particularly on the poleward side of the mid-latitude jet. The latitudinal gradient of the anomaly implies anomalous easterly forcing by the eddies poleward of the peak anomaly at $45^{\circ}$ and westerly forcing equatorward of the peak. Comparison with the zonal wind anomalies in Figure 3(b) reveals that these forcing regions coincide with wind anomalies of the same sign. The eddy forcing peaks in the upper troposphere, driving the zonal mean state away from thermal wind balance there. Balance is maintained by an anomalous zonally symmetric overturning circulation, whose Coriolis force balances the eddy forcing locally in the upper troposphere, and which yields a low level anomaly-balance between drag and 
a Coriolis force of the opposite sign to that in the upper troposphere. The streamfunction of the anomalous meridional circulation for run U5 (Figure 5(b)) coincides with the anomalous eddy forcing and zonal wind in precisely this manner.

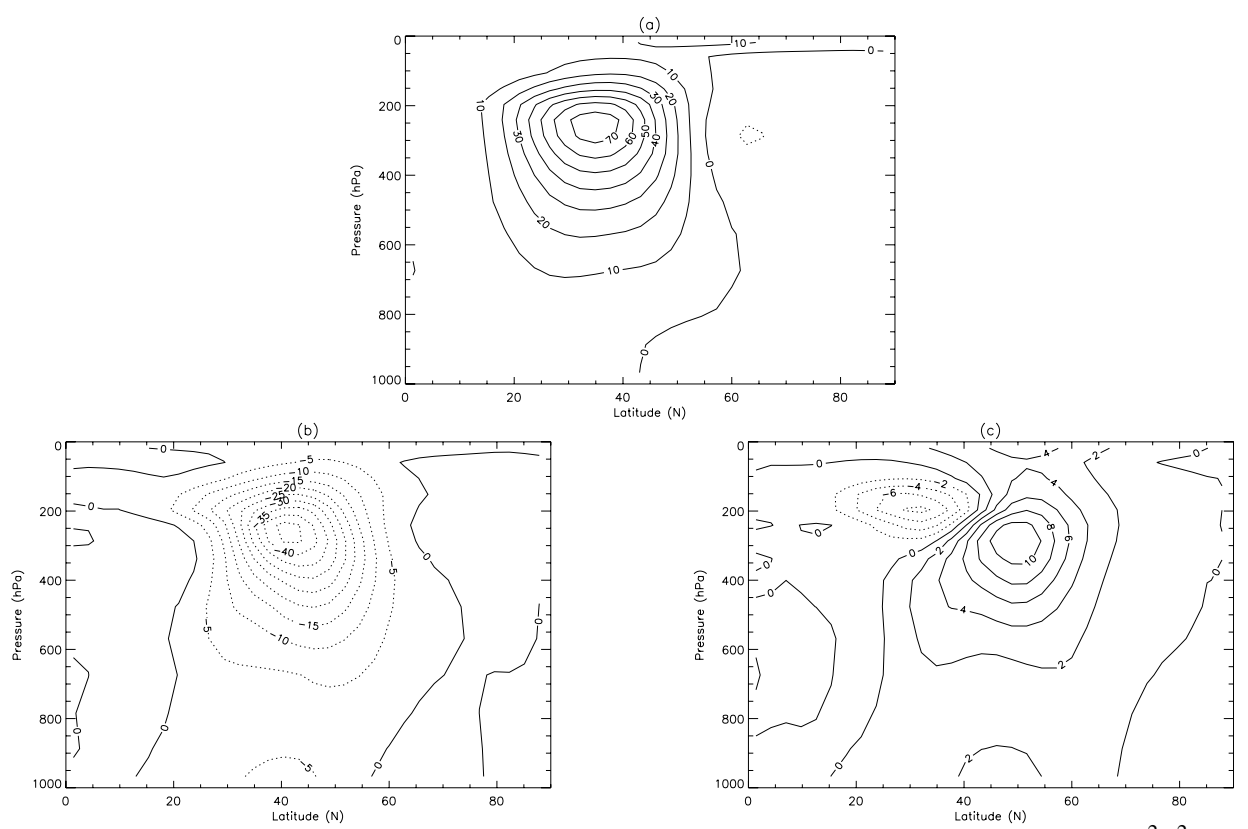

Figure 4 Poleward flux of westerly momentum by the zonally asymmetric eddies [ $\left.u^{\prime} v^{\prime}\right]\left(\mathrm{m}^{2} \mathrm{~s}^{-2}\right)$ for (a) the control run C; (b) the U5 - C anomaly and (c) the E5 - C anomaly. Average of 2 hemispheres.

The anomalous meridional circulation also affects the zonal mean temperature, by vertical advection of potential temperature in its vertical branches, in such a way as to maintain thermal wind balance in the presence of the eddy momentum forcing. This acts to increase the meridional temperature gradient below upper-level westerly eddy forcing and reduce it below easterly eddy forcing. The temperature field (not shown) reveals, for run U5, a warm anomaly poleward of $45^{\circ}$ coincident with the anomalous mean descent shown in Figure 5(b) and a cold anomaly equatorward of this latitude coincident with anomalous mean ascent. Thus a thermal perturbation applied only in the stratosphere produces vertical bands of warming and cooling in the troposphere.

To complete the picture, thermal forcing by the anomalous meridional circulation is balanced locally by a combination of anomalous convergence of the northward eddy heat flux and diabatic heating. The former comprises an equatorward shift and weakening of the poleward heat flux. The latter is inevitable in the sGCM experiments, in which all thermal anomalies in the troposphere are linearly damped by the Newtonian relaxation, since the tropospheric reference state is unchanged in the integrations. 


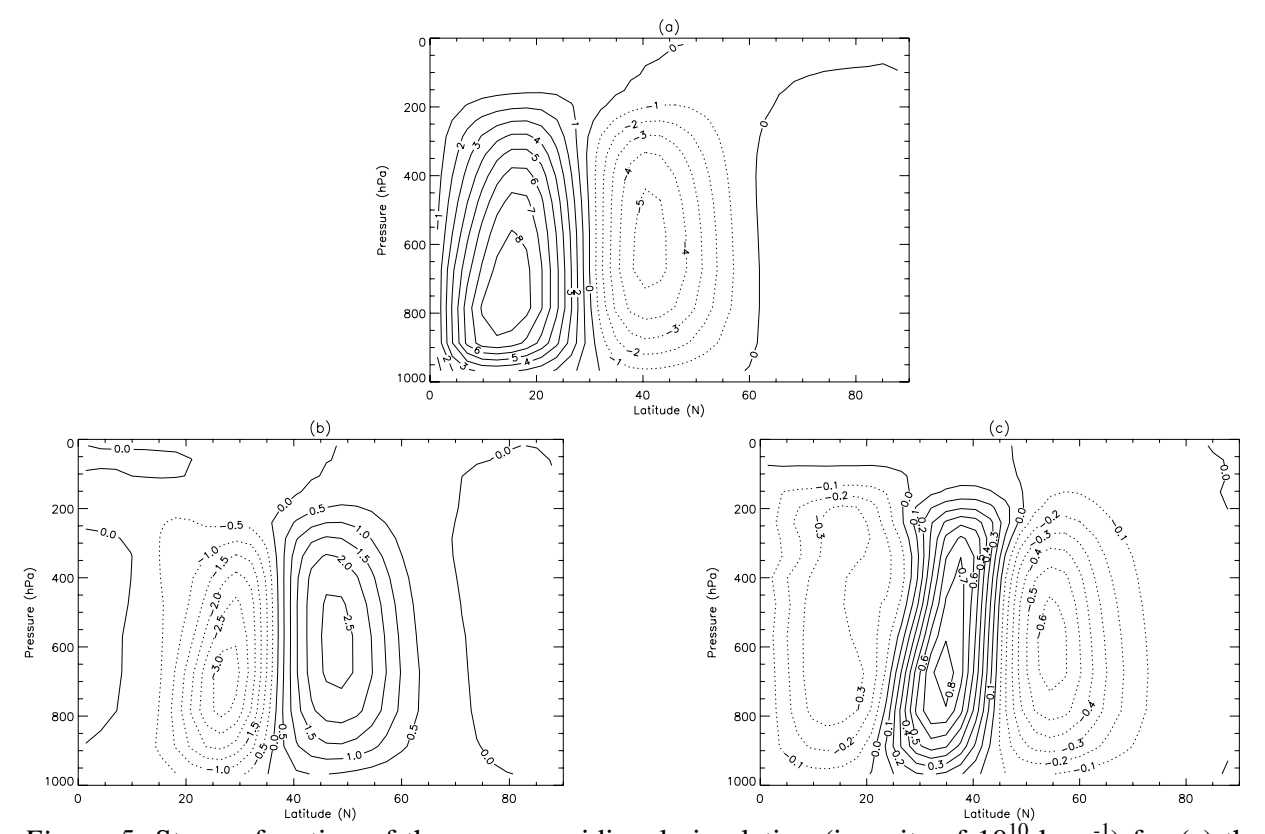

Figure 5 Stream-function of the mean meridional circulation (in units of $10^{10} \mathrm{~kg} \mathrm{~s}^{-1}$ ) for (a) the control run C; (b) the U5 - C anomaly and (c) the E5 - C anomaly. Average of 2 hemispheres.

A similar relationship between the various anomaly fields holds for experiment E5, although the anomalies are of smaller magnitude. The northward eddy momentum flux anomaly (Figure 4(c)) now has a dipolar structure, comprising a strengthening of the flux on the poleward flank of the jet and a weakening on its equatorward flank. This corresponds to a tripolar pattern of anomalous momentum flux convergence that coincides with the zonal wind anomalies in Figure 3(d). The low latitude change is larger, however, so that the net effect is to weaken the jet and displace it poleward. The anomalous meridional circulation (Figure 5(c)) locally counteracts the anomalous momentum forcing with its vertical branches again producing a response in tropospheric temperatures. In this case these consist of bands of cooling around $30^{\circ}$ and $65^{\circ}$ and a band of warming in the $40-55^{\circ}$ latitude region. This pattern is qualitatively very similar to the solar response found by Haigh (2003) in an analysis of NCEP Reanalysis temperatures.

For interpretation of the model experiments we find a useful diagnostic to be the vertically-integrated budget of zonal momentum. The rate of change of total westerly momentum integrated through a vertical column of atmosphere can be partitioned into components due to the zonally averaged flow, C_zonal, and the zonally asymmetric flow, C_eddy, with dissipation due to surface drag. Since the model's low-level flow is damped by the linear drag parameterization, latitudinal displacements in the westerly jet must be maintained by convergence of the poleward momentum flux anomaly C_total (= C_eddy + C_zonal). Terms in the 
vertically integrated momentum budget for the control experiment, shown in Figure 6(a), confirm that the surface stress is accurately balanced at each latitude by the convergence of the poleward flux of westerly momentum, C_total,, and that this is dominated by its eddy component. Both the mid-latitude surface westerlies and the easterly trade-winds are maintained by the poleward momentum flux. The divergence of the mean circulation term, C_zonal, is generally small and reflects the latitudinal structure of the upper-tropospheric branches of the Hadley and Ferrel cells. It opposes the eddy flux convergence in mid-latitudes and leads to a small displacement of the eddy flux convergence and surface stress in the sub-tropics.
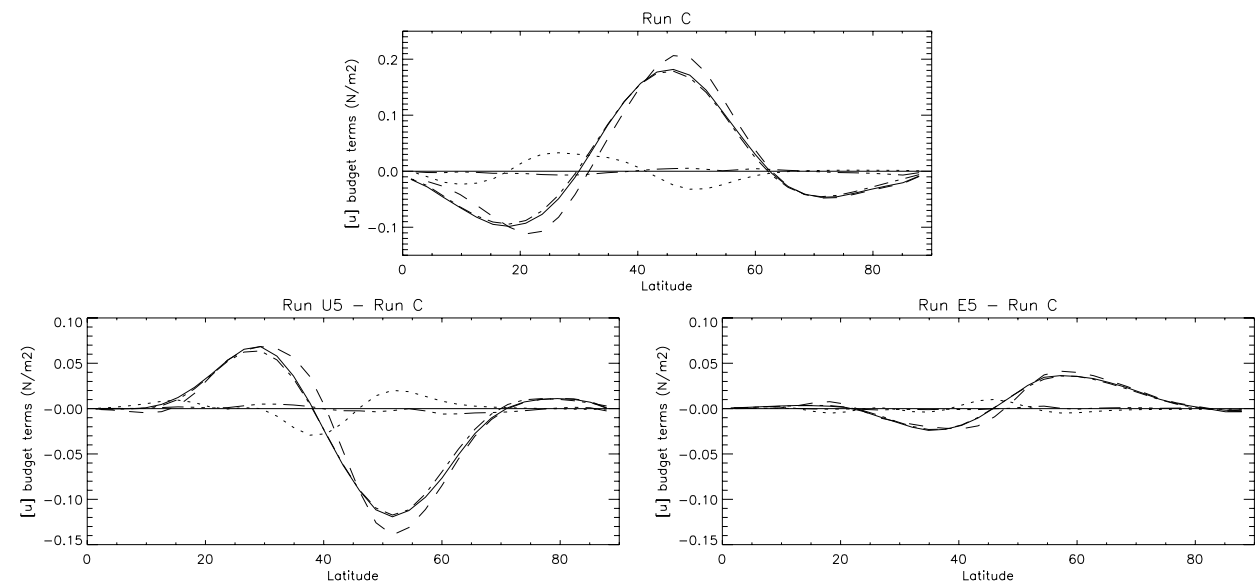

Figure 6 Terms in the vertically integrated budget of westerly momentum for (a) the control run C; (b) the U5 - C anomaly and (c) the E5 - C anomaly. Each panel shows the convergence of the flux of westerly momentum due to the zonally asymmetric eddies (dashed line), the zonally averaged flow (dotted) and their sum (solid line); the surface stress (dash-dotted) and the budget residual (dash-triple dotted). All quantities are in units of $\mathrm{Nm}^{-2}$.

The momentum budget anomalies in experiments U5 and E5, relative to the control, are shown in Figure 6(b) and (c). It is clear that the eddy flux convergence dominates the anomalies, balancing the surface stress dipole associated with the equatorward jet shift in U5 and the tripole associated with the poleward shift in E5. The mean circulation term is small in comparison, but accounts for a small displacement between the peak stress anomaly and peak eddy flux convergence anomaly on the equatorward side of the jet in each experiment. We conclude that the jet displacements in the model experiments are primarily maintained by the changes in poleward eddy momentum flux. The anomalous eddy flux convergence is concentrated in the upper troposphere, driving an anomalous mean circulation (displacement of the Ferrel cell) which effectively transfers the anomalous momentum forcing downwards, where it is balanced by the surface stress anomaly. 


\section{Investigation of mechanisms using an ensemble of model spin-ups}

We now ask to what extent the diagnostic picture presented in the preceding section can be interpreted causally, beyond the statement that the thermal perturbations imposed in the stratosphere lead to the modelled changes in the troposphere. All the experiments involve long-term averaged steady-states, in which the anomalous mean flow and eddy forcing terms in the budget equations must balance. The vertically integrated momentum balance immediately implies that latitudinal displacements of the jets necessarily involve up-gradient anomalous momentum fluxes, which we have found to be dominated by the eddy component, while thermal wind balance implies that the baroclinic component of such displacements will be associated with anomalous temperature gradients.

To investigate the chain of causality in the establishment of the equilibrium response an experiment has been conducted in which, starting from fields produced by the control run C, the thermal perturbation used in run U5 is suddenly switched on and the time-evolving state monitored. The results from a single such spin-up experiment would be sensitive to the initial conditions and subject to the effects of model internal variability so could not be statistically representative. To counteract this problem an ensemble of 200 runs has been carried out. In each of these the same perturbation is applied but the initial conditions are set from points 50 days apart in the control run. Each member of the ensemble was run for 50 days and output produced daily. In the figures that follow all the plots show results which are averaged over the 200 members of the ensemble and over the two hemispheres (with hemispherically asymmetric fields scaled appropriately).

Figure 7 shows how the zonal mean zonal wind, horizontal eddy momentum flux and mean meridional circulation evolve. Comparison with Figures 3(b), 4(b) and 5(b) respectively reveals that by days 40-49 the spin-up fields have only progressed a small fraction of the way towards the equilibrium state but are already showing some of the features of the equivalent equilibrium responses: the zonal wind has the characteristic weakening and equatorward shift of the jet while the eddy momentum fluxes and mean meridional circulation are both weakened. There are also some clear differences between the patterns of the time-evolving and equilibrium responses, however, which give an indication of how the state develops. In the spinups the signal is initially concentrated in the stratosphere with effects in the troposphere lagging behind. The peak (negative) anomaly in zonal wind by days 40-49 (approximately $-1.75 \mathrm{~ms}^{-1}$ ) occurs near $45^{\circ}, 100 \mathrm{hPa}$ while that in the equilibrium response $\left(12 \mathrm{~ms}^{-1}\right)$ is at $50^{\circ}, 250 \mathrm{hPa}$. The positive anomaly at lower latitudes (representing the equatorward shift of the jet) and the signals extending down through the troposphere are barely established. 

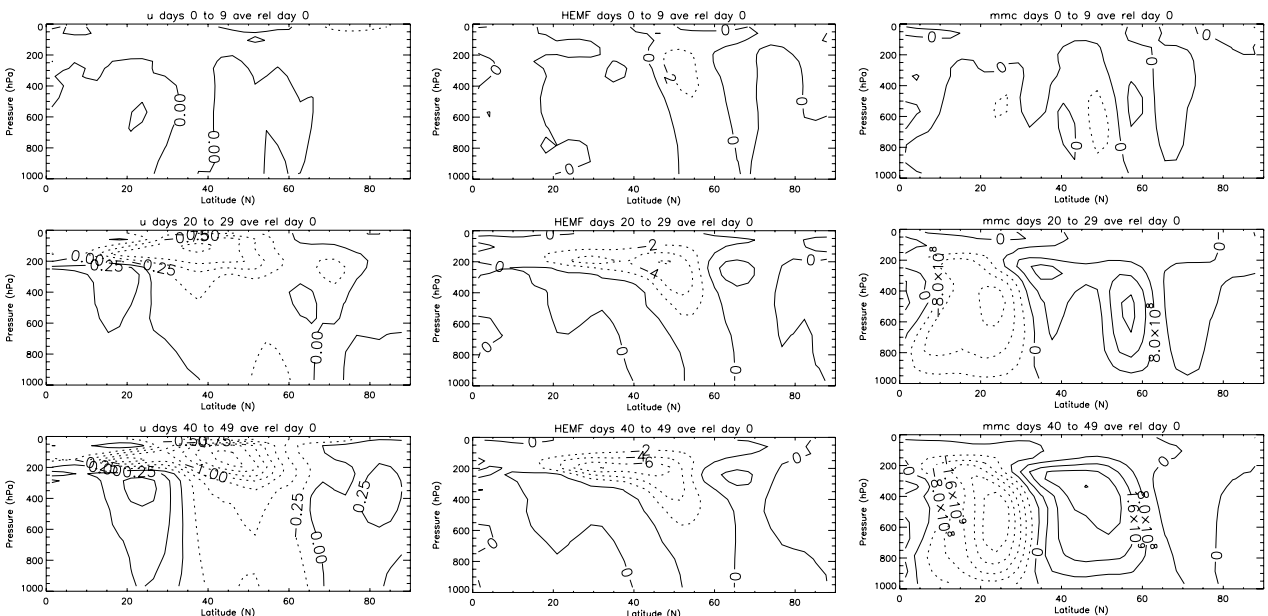

Figure 7 10-day averages of fields (presented as the anomaly from the initial state) evolving after a switch-on of the U5 perturbation at day 0. Top: days 0-9; Middle: days 20-29; Bottom: days 4049. Left: Zonal mean zonal wind $\left(\mathrm{m} \mathrm{s}^{-1}\right)$; Centre: Poleward flux of westerly momentum by the zonally asymmetric eddies $\left(\mathrm{m}^{2} \mathrm{~s}^{-2}\right)$; Right Stream-function of the mean meridional circulation $(\mathrm{kg} \mathrm{s}$ $\left.{ }^{1}\right)$.

This effect is seen, perhaps even more clearly, in the eddy momentum fluxes. By days 40-49 of the spin-up there is a clear negative anomaly but it is almost exclusively confined to the tropopause region. There is some evidence, however, of a signal propagating downwards into the troposphere, strengthening with time. The mean meridional circulation anomaly extends through the troposphere but is more weighted towards the upper troposphere than in the equilibrium case.

Kushner and Polvani (2004) have used a similar methodology to investigate high latitude stratosphere-troposphere interaction associated with annular variability and showed that the zonal wind anomaly takes about 500 days to equilibrate in the troposphere. They also used a zonally symmetric version of their simplified GCM in attempt to diagnose the roles of tropospheric and stratospheric eddies on the equilibrium response. They found that stratospheric eddy-driving induces a response in the troposphere but without feedback from the tropospheric eddies the full equilibrium response in the troposphere could not be reproduced.

Our results show that heating the lower stratosphere reduces the wave fluxes in the tropopause region by lowering the tropopause, and leads to coherent changes developing in the troposphere. In Section 3 we found, by analyzing the vertically integrated budget of zonal momentum for the equilibrium experiments, that the displacements of the jets were primarily maintained by the changes in poleward eddy momentum flux. Now we investigate if the time evolution of the vertically integrated momentum budget from the spin-up experiment can provide an indication of the chain of causality in the observed effects. Figure 8 shows 10-day averages of the momentum budget components as deviations from the initial state. These can be compared with Figure 6(b) which presented the values for the U5 equilibrium run. 

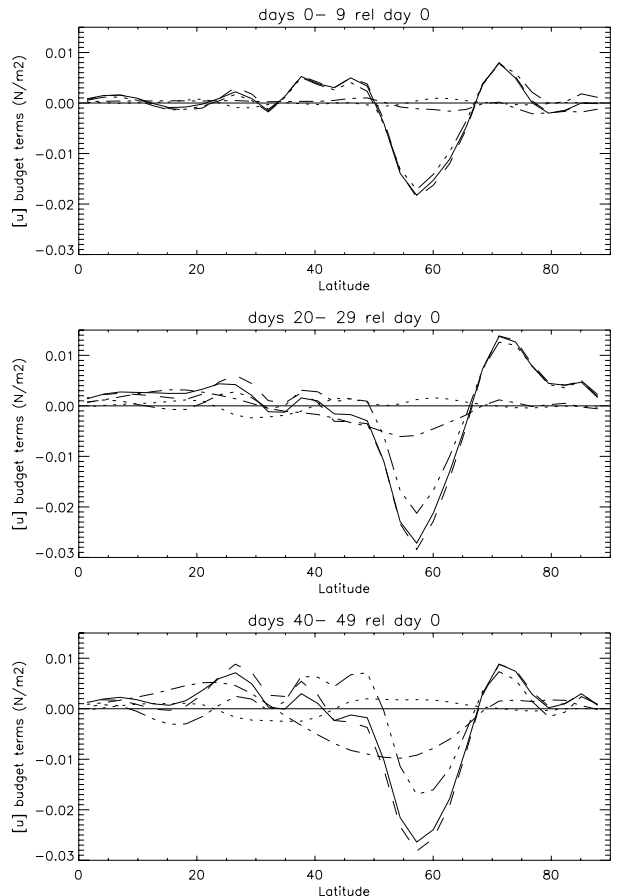

Figure 8 10-day averages of terms in the vertically integrated budget of westerly momentum (presented as the anomaly from the initial state) evolving after a switch-on of the U5 perturbation at day 0 . Each panel shows the convergence of the flux of westerly momentum due to the zonally asymmetric eddies (dashed line), the zonally averaged flow (dotted) and their sum (solid line); the surface stress (dash-dotted) and the budget residual (dash-triple dotted). All quantities are in units of $\mathrm{Nm}^{-2}$.

The eddy term in the spin-up shows a developing negative anomaly around 50$65^{\circ}$ similar (though smaller in magnitude) to that in the equilibrium case. The positive anomaly in the subtropics is less well developed, however and the anomalies in the zonally averaged term are shifted towards the equator. Consistent with the latter, in the spin-up case the surface stress term is not balancing the net momentum flux but is lagging behind suggesting that the anomalous Ferrel cell driven by the eddies has not become sufficiently well established to transport momentum anomalies down to the surface. This provides a clear indication that the chain of causality starts with modifications to the wave flux convergence at the tropopause. 


\section{Conclusions}

We conclude from our equilibrium experiments that imposed changes in the lower stratospheric temperature forcing lead to coherent changes in the latitudinal location and width of the mid-latitude jetstream and its associated storm-track, and that eddy / mean-flow feedbacks are crucial to these changes. Imposed stratospheric warming and an associated lowering of the tropopause tends to weaken the jet and storm-track eddies; equatorial stratospheric warming displaces the jet polewards while uniform warming displaces it markedly equatorwards.

We suggest that the observed climate response to solar variability is brought about by a dynamical response in the troposphere to heating predominantly in the stratosphere. The effect is small, and frequently masked by other factors, but not negligible in the context of the detection and attribution of climate change. The results also suggest that, at the Earth's surface, the climatic effects of solar variability will be most easily detected in the sub-tropics and mid-latitudes.

Details of the mechanisms involved in the transfer of the effects of the stratospheric forcing to the troposphere are still not clear. However, our analysis of the vertically integrated momentum budget in the ensemble of spin-up runs shows that changes in the static stability of the tropopause region initially cause a weakening of eddy momentum flux convergence and that the transfer of the momentum anomaly by the mean circulation to the surface, where it is dissipated, lags behind. This suggests that understanding the effects of the stratospheric perturbations on wave propagation will provide the key to unraveling the mechanisms of how a solar (or indeed any other) influence in the stratosphere may influence tropospheric climate.

\section{References}

Crooks, S. and Gray, L. J.: 2005, 'Characterisation of the 11-year solar cycle using a multiple regression analysis of the ERA-40 dataset'. J. Clim., 18, 996-1015.

Gleisner, H. and Thejll, P.: 2003, 'Patterns of tropospheric response to solar variability'. Geophys Res Lett, 30, art. no. 1711.

Haigh, J. D.: 1996, 'The impact of solar variability on climate'. Science, 272, 981-984.

Haigh, J. D.: 1999, 'A GCM study of climate change in response to the 11-year solar cycle'. Quart. J. Roy. Meteorol. Soc., 125, 871-892

Haigh, J. D.: 2001, Climate variability and the role of the Sun. Science, 294, 2109-2111

Haigh, J. D.: 2003, 'The effects of solar variability on the Earth's climate'. Phil. Trans. Roy. Soc A., 361, 95-111.

Haigh, J. D., Blackburn, M. and Day, R.: 2005, 'The response of tropospheric circulation to perturbations in lower stratospheric temperature'. J. Clim., 18, 3672-3691.

Haynes, P. H.: 2005, 'Stratospheric dynamics'. Ann. Rev. Fluid Mech., 37, 263-293.

Held, I. M. and Suarez, M. J.: 1994, 'A proposal for the intercomparison of the dynamical cores of atmospheric general circulation models’. Bull. Amer. Meteor. Soc., 75, 1825-1830.

Hoskins, B. J. and Simmons, A. J.: 1975, 'A multi-layer spectral model and the semi-implicit method’. Quart. J. Roy. Meteorol. Soc., 101, 637-655. 
Kushner, P. J. and Polvani, L. M.: 2004, 'Stratosphere-troposphere coupling in a relatively simple AGCM: the role of eddies'. J. Climate, 17, 629-639.

Larkin, A., Haigh, J. D. and Djavidnia, S.: 2000, 'The effect of solar UV irradiance variations on the Earth's atmosphere'. Space Science Reviews, 94, 199-214

Matthes K., Langematz U., Gray, L. J., Kodera, K. and Labitzke, K: 2004, 'Improved 11-year solar signal in the freie universitat Berlin climate middle atmosphere model (FUB-CMAM)'. $J$. Geophys. Res., 109, D06101.

Polvani, L. M. and Kushner, P. J.: 2002, 'Tropospheric response to stratospheric perturbations in a relatively simple general circulation model’. Geophys. Res. Lett., 29, 10.1029/2001GL014284.

Rind, D. and Balachandran, N. K.: 1995, 'Modeling the effects of UV variability and the QBO on the troposphere-stratosphere system. Part II: the troposphere’. J. Clim., 8, 2080-2095.

Shindell, D., Rind, D., Balachandran, N., Lean, J. and Lonergan, P.: 1999, 'Solar cycle variability, ozone, and climate', Science 284, 305-308.

Simmons, A. J. and Burridge, D. M.: 1981, 'An energy and angular-momentum conserving vertical finite-difference scheme and hybrid vertical coordinates’. Mon. Wea. Rev., 109, 758766.

van Loon, H. and Shea, D.: 2000, 'The global 11-year signal in July-August'. Geophys. Res. Lett., 27, 2965-2968 